\section{Enzyme electrophoresis}

Handbook of Enzyme Electrophoresis in Human Genetics. By Harry Harris and D. A. Hopkinson. (North-Holland : Amsterdam, 1976.)

RECOGNITION that enzymes may occur in multiple, electrophoretically distinct, forms first found important practical applications in clinical laboratories where attention has been concentrated on the relatively few isoenzymes known to be of diagnostic significance. The discovery of isoenzymes, however, has had an even greater impact in genetics. Enzyme heterogeneity has been studied by electrophoretic and other techniques at the MRC Human Biochemical Genetics Unit for about two decades. The authors have now provided a distillate of their vast experience in this handbook which is supplied as loose leaves in a ring binder to which supplements can be added as they become available.

After briefly outlining the significance of isoenzymes, the principles of enzyme electrophoresis and methods for their subsequent detection, techniques for the more important methods, especially starch-gel edectrophoresis, are described in sufficient detail to enable them to be set up elsewhere. The main part of the work is devoted to individual enzymes with summaries of necessary technical modifications and examples of the patterns obtained. Finally, brief tabulated sections deal with enzyme subunits and the effects of thiol reagents, together with useful appendices.

As would be expected from such distinguished authors, the work is authoritative and well written. The methods are clearly and concisely presented and, although no attempt is made to give a complete bibliography, sufficient key references are included to enable original sources to be traced. Minor criticisms concern the use of certain obsolescent terminology-for example, glutamate-oxaloacetate transaminase-and the lack of standardisation of the abbreviations. These, however, are trivial matters which do not detract from the value of the book. It will be useful, not only to geneticists, but also to clinical biochemists who at any time may encounter hitherto unsuspected enzyme anomalies.

J. H. Wilkinson

J. H. Wilkinson is Professor of Chemical Pathology at Charing Cross Hospital Medical School, University of London, UK.

\section{Subnuclear components}

Subnuclear Components: Preparation and Fractionation. Edited by G. D. Birnie. Pp. 334. (Butterworth: London and Boston, Massachusetts, March 1976.) £15.

Subnuclear Components is a valuable book for the practising research worker. It explains the immediate reasoning behind some popular preparative techniques in an area in which the end justified the means. Happily, assessment of the end-product is included in most of the chapters. The discussions all lie in the areas between detailed descriptions of techniques and expositions of general principles, and provide a very valuable insight into the thinking and background associated with some important experimental procedures. Each of the eight chapters is written by a different author or group of authors, all of whom are busy research workers themselves; and it is not surprising therefore that the text and proof reading are not as polished as I would otherwise expect for $£ 15$ per copy. Less excusable is the long gap between writing and publication, during which time some material has been left behind by events such as the nucleosome model for chromatin; and quite crazy is the tiny print used for the figure captions. Although many of the main techniques have been covered there are gaps in such areas as ribonucleoprotein particles, mitotic apparatus and DNA fractionation (DNA preparation is well covered in a chapter by $\mathrm{Dr}$ Butterworth). In the best chapters the available methods are critically discussed and a few chosen for detailed analysis. There are, however, some important gapsfor example, in the otherwise excellent discussion of histone preparation, chromatographic methods are almost ignored. Such problems are inevitable when the authors are presenting the detailed 'behind-the-scenes' analysis which make Subnuclear Components so valuable.

Harry R. Matthews

Harry R. Matthews is a senior lecturer in the Department of Physics at the Portsmouth Polytechnic, UK

\section{Protoplasmic connections in plants}

Intercellular Communication in Plants: Studies on Plasmodesmata. Edited by B. E. S. Gunning and A. W. Robards. Pp. xvi+387. (Springer: Berlin and New York, 1976.) DM72; \$29.60.

THIS volume contains 14 review papers presented and discussed at a meeting held in Canberra in June 1975. The aim of the volume according to the editors is to summarise what is known about the nature of plasmodesmata and assess the significance of these intercellular protoplasmic connections in plant physiology. The contributors have performed a valuable service in collecting together information which was previously scattered widely in the literature, and the editors are to be congratulated on the skill with which they have integrated the papers and summarised the discussions at what must have been a very lively meeting.

It is now nearly 100 years since Tangl described strands of protoplasm traversing the cell walls in the endosperm of certain seeds, but because of their extreme fineness $(30-60 \mathrm{~nm})$ their very existence remained in some doubt until the advent of the electron microscope. As Robards points out in this volume, in spite of intensive study in the past 10 years, many of the details of struc- ture remain unresolved. In particular the relationship between the desmotubule and the endoplasmic reticulum of the adjoining cells is still uncertain.

Even greater controversy surrounds the operation of plasmodesmata and much of the volume is devoted to this problem. The early suggestions of Haberlandt that protoplasmic connections may function both in the translocation of substances and transmission of stimuli are supported by a wealth of more recent circumstantial evidence, but an unequivocal demonstration that things actually move through plasmodesmata is still awaited. Tyree has established that plasmodesmata may have sufficient capacity to transport solutes at the fluxes observed, but the basis of such transport is still obscure. This volume, with its extensive bibliography, will form an excellent springboard for anyone wishing to advance our knowledge in an intriguing field of research.

The book is printed from 'cameraready' typescripts without justified margins and one might have expected that the saving in time and cost would be reflected in more rapid publication and lower price than has been achieved.

James F. Sutcliffe

James Sutcliffe is Professor of Plant Physiology in the School of Biological Sciences at the University of Sussex, $U K$, and Editor-in-Chief of Annals of Botany. 